\title{
A RARE CASE: AMEGAKARYOCYTE TROMBOCYTOPENIA AS EARLY MANIFESTATION OF JUVENILE SYSTEMIC LUPUS ERYTHEMATOSUS
}

\author{
Yeni Amalia $^{1 *}$ Wisnu Barlianto Susanto Nugroho ${ }^{2}$ \\ ${ }^{1}$ Faculty of Medicine, University of Islam Malang \\ ${ }^{2}$ Faculty of Medicine, Universitas Brawijaya
}

\begin{abstract}
BACKGROUND Systemic lupus erythematosus (SLE) is a chronic autoimmune disease which results in inflammation and eventual damage in a broad range of organ systems. Trombocytopenia is a common complication in patients with SLE, but amegakaryocyte trombocytopenia (AMT) is a rare disorder in SLE that characterized by isolated thrombocytopenia as a consequence of a marked decrease in or total absence of megakaryocytes in an otherwise normal bone marrow.
\end{abstract}

CASE Lu, a wellnourished 13 y.o girl, complaining for high fever for 2 weeks, diminished body weight, artralgia, furunculosis, oral apthae and palour for 1 year, and bruising extremities for 2 motnhs. There was hepatosplenomegali.

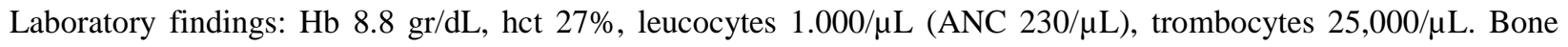
marrow puncture revealed amegacaryocyte thrombocytopenia, absence of megacaryocyte while erythropoesis and granulopoesis were normal. Anti ds DNA $223.8 \mathrm{iu} / \mathrm{ml}(+)$; ANA test 101.7 unit (+), microalbumin urine $10 \mathrm{mcg} / \mathrm{mg}$ (-), C3 complement $40 \mathrm{mg} / \mathrm{dL}(\downarrow)$, ACA IgM $20.6 \mathrm{U} / \mathrm{ml}(+)$, ACA IgG $17.3 \mathrm{U} / \mathrm{mL}(+)$. The case fulfilled 5 criteria of ACR for SLE. The patient administered ceftazidim and gentamycin for febrile neutropenia, and high dose methylprednisolon injection for SLE.

CONCLUSION A rare case of amegakaryocyte thrombocytopenia in juvenila SLE has been successfully diagnosed. Key word: child, girl, SLE, AMT

*correspondence author:

dr. Yeni Amalia, M. Biomed., Sp.A

Fakultas Kedokteran Universitas Islam Malang Jl. MT Haryono 193 Malang

Email: yeniamalia@unisma.ac.id

\section{INTRODUCTION}

Systemic lupus erythematosus (SLE) is a severe, chronic autoimmune disease which results in inflammation and eventual damage in a broad range of organ systems. SLE is a relatively rare disease in childhood with an estimated incidence ranging from 10-20/ 100,000 children, depending on ethnicity. Childhood SLE affects girls more often than boys, and it can occur at any 
age, SLE becomes more frequent after five years of age and is increasingly prevalent after the first decade of life. ${ }^{1-3}$

Trombocytopenia is a common complication in patients with SLE, and its prevalence ranges from $7-30 \%$. The two main mechanisms of thrombocytopenia in SLE are increased platelet destruction mediated by antiplatelet autoantibodies, similar to the mechanism seen in immune thrombocytopenic purpura (ITP), and impaired thrombopoiesis, due to the absence of or a decreased number of megakaryocytes in the bone marrow. The latter disorder is also known as amegakaryocytic thrombocytopenia (AMT). AMT is a rare disorder characterized by isolated thrombocytopenia that is often severe (platelet count $\left.<20-30 \times 10^{9} / \mathrm{L}\right)$, as a consequence of a marked decrease in or total absence of megakaryocytes in an otherwise normal bone marrow. ${ }^{4,5}$

We report a rare case of AMT in 13 year-old girl with SLE that mimicked malignancy. The aim of presenting this case is to increase awareness of this condition since it is not generally considered as SLE.

\section{CASE REPORT}

Lu, a 13 year-old wellnourished girl was referred for suspected Acute Leukemia. She had suffered from a high fever since 14 days before admission and often got relapsing fever for 1 year. There were history of palour, weakness, recurrent oral apthae and furunculosis, and arthralgia for 1 year. There was also recurrent bruising extremities for 2 months. From referreal hospital there was history of pancytopenia and febrile neutropenia. The patient had administered with Ceftazidim and PRC transfusion. In addition there was no history of malignancy, blood diseases, or other chronic diseases such as tuberculosis or hepatitis in her familiy. She got menarche 1 month before admission.

She looked alert, moderately ill, with fever (axillary temperature $38^{\circ} \mathrm{C}$ ). There was oral ophthae, enlargement of the liver 1/3-1/3 sharp edge, enlargement of the spleen Schuffner 1, bruising and furuncle on the thigh. The other physical examination were normal.

Initial laboratory findings showed hemoglobin $8.8 \mathrm{gr} / \mathrm{dL}$, Hematocrites 27\%, leucocytes $1.000 / \mu \mathrm{L} \quad(\mathrm{ANC} \quad 230 / \mu \mathrm{L}), \quad$ trombocytes $25,000 / \mu \mathrm{L}$, normal renal function test (ureum $19.7 \mathrm{mg} / \mathrm{dl}$, creatinine $0.3 \mathrm{mg} / \mathrm{dl}$ ), normal liver function test (AST $20 \mathrm{U} / \mathrm{L}$, ALT $25 \mathrm{U} / \mathrm{L}$ ), CRP $1.53 \mathrm{mg} / \mathrm{dl}$. Urineanalysis, and coagulation function were normal. The anteroposterior chest $\mathrm{X}$-ray was normal, and the abdominal ultrasound was revealed hepatosplenomegali. ECG was normal.

Based on the history taking, physical examination and laboratory findings, the patient was suspected for malignancy. She then underwent Bone Marrow Aspirations (BMA) that revealed normocellularity with good erytropoesis and granulopoesis activities, and diminished megakaryocyte activity (figure1). Fe storage was positive and her Coomb's test was negative. 

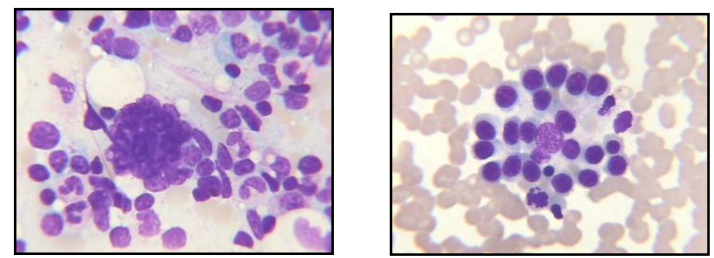

Figure1. Bone Marrow Aspiration revealed amegakaryocyte thrombocytopenia (AMT)

Based on recent supportive examinations, pancytopenia due to amegakaryocytic thrombocytopenia due to Systemic Lupus Erythematosus with differential diagnosis infection were diagnosed.

The laboratory finding performed later indicated a sterile urine culture, $\mathrm{HbsAg}(-)$; anti HCV (-); IgM Salmonella (-); Anti dsDNA 223.8 iu/ml (positive > 92.6 IU/mL); ANA test 101.7 unit (+). The next supportive examination revealed microalbumin urine $10 \mathrm{mcg} / \mathrm{mg}(\downarrow), \mathrm{C} 3$ complement $40 \mathrm{mg} / \mathrm{dL}(\uparrow)$, ACA IgM $20.6 \mathrm{U} / \mathrm{ml}$ (+), ACA IgG $17.3 \mathrm{U} / \mathrm{mL}(+)$. The findings fulfilled 5 criteria of ACR for SLE.

The patient was given intravenous methyl prednisolon $1 \times 80 \mathrm{mg} \quad(2 \mathrm{mgs} / \mathrm{kgBW} /$ day $)$, antibiotics were continued until ANC $>500 / \mu 1$ with ceftazidim and gentamycin due to prolonged febrile neutopenia. She administered sunblock cream, nutritional diet as many as RDA (2500 kkal), and vitamin D s to prevent osteoporosis.

On the $11^{\text {th }}$ day of admission she got better, no fever, improvement of oral apthae and furuncle. The complete blood count revealed $\mathrm{Hb} 11.4$ $\mathrm{md} / \mathrm{dL}$, leucocyte $2900 \mathrm{~mm}^{3}, \mathrm{PCV}$, trombocytes $26,000 / \mathrm{mm}^{3}$, ANC 870 . The administration of injected antibiotics were stopped and changed to oral ones.

\section{DISCUSSION}

SLE in children affects girls more often than boys, and becomes more frequent after five years of age and is increasingly prevalent after the first decade of life. In this case, SLE was diagnosed in a 13 year-old girl who had just entered puberty. ${ }^{1-}$ 3

The patient was refered from a private hospital for suspected acute leukemia. Constitutional symptoms such as fatigue, fever and weight loss, with organomegali (hepatosplenomegali) and pancytopenia are very common in malignancy. ${ }^{1-}$ ${ }^{3,6,7}$ Presentation of pancytopenia is alarming and indicates the possibility of either a bone marrow failure syndrome or acute malignancy particularly when associated with lymphadenopathy or organomegaly. Aplastic anemia and ALL are the most common and the most dangerous established causes of pancytopenia. Acute leukemia itself may mimic initially as aplastic anemia. ${ }^{7}$

The patient underwent a Bone Marrow Aspiration (BMA) procedure that revealed amegakaryocyte thrombocytopenia (AMT. It can occur alone as an idiopathic entity; in association with lymphoproliferative disorders, SLE, and other autoimmune diseases, infections, solid tumors, B12 deficiency, and ethanol abuse or after exposure to drugs. AMT can also be the initial manifestation of bone marrow disorders such as myelodysplastic syndromes or aplastic anemia. Due in part to its rarity and in part to its 
heterogeneous nature, the pathogenetic mechanisms of this disorder have not been well defined; in fact, its etiology is likely to be diverse. $4,5,8$

The laboratory finding performed later showed a sterile urine culture, Anti dsDNA 223,8 $\mathrm{iu} / \mathrm{ml}(+)$; ANA test 101,7 unit (+), C3 complement 40 mg/dL ( $\downarrow)$, ACA IgM 20.6 U/ml (+), ACA IgG $17.3 \mathrm{U} / \mathrm{mL}(+)$. Based on these findings, the patient fulfilled 5 criteria for ACR for SLE, thus the AMT was due to SLE. (Table $1)^{9}$

SLE is a multisystem disorder with wideranging clinical and laboratory manifestations. Of these, thrombocytopenia is a common finding with platelet counts less than $100 \times 10^{9 / \mathrm{L}}$ with a prevalence that ranges from $8-30 \%$ of the patients. The purported mechanism leading to thrombocytopenia in SLE includes antiGPIIb/IIIa antibody-mediated platelet destruction and inhibition of megakaryopoesis by antibodies directed against the TPOR (cMpl). Subsequent studies have suggested that AMT can develop as a consequence of cell-mediated inhibition or destruction of megakaryocytes, possibly by monocytes or a clonal population of $\mathrm{T}$ lymphocytes. These findings, in particular, suggest that thrombocytopenia in SLE may overlap with AMT. Alternatively, AMT may simply be an extreme manifestation of immunemediated thrombocytopenia in SLE. A wide range of therapies have been used in patients with AMT, including conventional immunosuppressive agents suc as steroid, anti- thymocyte globulin, cyclosporine A, a thrombopoietin (TPO) nonpeptide mimetic (rituximab), immunomodulators such as intravenous immunoglobulin (IVIG), splenectomy, and allogeneic bone marrow transplant. The evidence for each of these is weak, and based solely on anecdotal evidence. Supportive therapy involves platelet transfusions to reduce the risk of intra-cranial hemorrhage and other bleeding manifestations, so did in this patient. ${ }^{4,5,8}$

The patient suffered from febrile neutropenia. Patients with a low ANC and prolonged neutropenia (eg, >10 days) are at further increased risk of infection. In this case, the patient suffered from severe febrile neutropenia lasted more than 10 days. The history of recurrent furunculosis and oral apthae tend to be related to this condition, since lower the ANC, the greater possibility of getting an infection. With the improvement of the ANC, the furucle, oral apthae and the fever also diminished. Based on the algorithm for the management of febrile neutropenia, the patient was administered single antibiotic injections (ceftazidim) for 5 days, then administered double antibiotic injections with aminoglicoside gentamicyn. Although the fever had subsided, the ANC was still $<500$ on the day 7 of neutropenia, thus the administration of antibiotics was continued until the ANC reached $>500 / \mu \mathrm{l}$. The ANC values had improved and reached $>500 / \mu 1$ on $11^{\text {th }}$ day admission, but because the neutropenia still persisted, the 
antibiotic was conticoued with oral administration. ${ }^{10-13}$

The etiology of SLE is unknown but genetic, environmental, and host immunologic factors are believed to interact and lead to disease expression. Autoantibody production is the hallmark of this disease with the mechanism leading to loss of self-tolerance and some evidence suggests that immune complexmediated complement activation is important in the renal and vascular damage of SLE. The association between HLA alleles and the level of various autoantibodies has also been studied. Anti-Ro and anti-La are powerfully associated with DR3/DR2 heterozygotes at the DQ alleles. Anti-Sm and anti-nRNP tend to be associated with alleles on haplotypes containing a DR4 or a DQ3. Various viruses are known to be associated with SLE, including myxoviruses, reoviruses, measles, and rubella and ebstein-barr virus. The other environmental factors such as exposue to silica, mercury, pesticides, and drugs are also associated with SLE. In this case the etiology of the SLE was still unclear. There was no history of silica, mercury, pesticide and also drug exposure in this patients. The possibility of previous viral infection such as EB virus

\section{Tabel 1. ACR crtiteria for $\mathrm{SLE}^{9}$}

\section{Malar rash}

2.Discoid rash

3.Photosensitivity

4.Oral ulcers

5.Arthritis

6.Serositis

(a)Pleuritis
(b)Pericarditis

7.Renal disorder

(a)Proteinuria $>0.5 \mathrm{~g} / 24 \mathrm{~h}$ or

$3+$,persistently

(b)Cellular casts

8.Neurological disorder

(a)Seizures

(b)Psychosis(having excluded other causes - for example, drugs)

9.Haemolytic disorder

(a)Haemolytic anaemia

(b)Leucopenia or $<4.0 \times 10^{9} / \mathrm{L}$ on

two or more occasions

(c)Lymphopenia or $<1.5 \times 10^{9} / \mathrm{L}$ on two or more occasions

(d)Thrombocytopenia $<100 \times 10^{9}$

$/ \mathrm{L}$

10.Immunologicaldisorders

(a)Raisedanti-

nativeDNAantibodybinding

(b)Anti-Smantibody

(c)Positivefindingofantiphospholipid antibodiesbasedon:

(i) $\mathrm{IgG} / \mathrm{M}$ anti cardiolipin antibodies

(ii)Lupus anticoagulant

(iii)False positive serological

test for syphilis, present for at least 6months

11.Antinuclear antibody in raised titre

A person shall be said to have SLE if four or more of the 11 criteria are present, serially or simultaneously, during any interval of observation.'

Revised criteria of the American College of Rheumatology for the

classification of SLE (modified from Hochberg, ArthritisRheum

1997;40:1725-34)

and the other virus as remaining possibility, but could not be prooved. The only environmental exposure she got was from sunlight. The genetic and immunologic susceptibility was perhaps were the main etiology of the SLE in this patient. ${ }^{1-3,9,14-}$ 16

The treatment for most manifestations of SLE does not differ among adults, children, and 
adolescents. However, children and adolescents with SLE have unique problems related to growth and development that affect both the need for and the impact of aggressive therapy. If only skin and joints are affected, treatment with non steroidal anti-inflammatory drugs alone or in combination with hydroxyl chloroquine may be sufficient. Because most children who have SLE have at least one major organ system involved, they usually require treatment with corticosteroids. Low-dose prednisone $(<0.5 \mathrm{mg} / \mathrm{kg} /$ day $)$ may be used for pleuritis as well as for persistent fever, skin involvement, and severe constitutional symptoms. Major organ disease (eg, diffuse proliferative glomerulonephritis) necessitates the use of high-dose prednisone. The initial dose is $2 \mathrm{mg} / \mathrm{kg}$ perday (maximum 60 to $80 \mathrm{mg}$ ), a minimum of 3 to 4 weeks followed by consolidation and then a gradual decrease in dosage. Immunosuppressive medications are used for treating people with more severe manifestations of SLE, such as damage to internal organ. Other agents are used for SLE treatment are immunosuppressive medications include methotrexate, azathioprine, cyclophosphamide, chlorambucil, and cyclosporine. This patient was administered with methylprednisopolon 2 $\mathrm{mg} / \mathrm{kg} / \mathrm{day}$, due to bone marrow failure (AMT). ${ }^{1-}$ $3,9,14,15,17,20$

The prognosis for children and adolescents with SLE who receive appropriate care is generally very good and presently, patients with SLE have a far better prognosis than 40 years ago. The ten-year survival rate is now estimated to be $80 \%-90 \%$. The survival rate for children and adolescents with SLE is nearly $100 \%$ at 5 years and $85 \%$ at 10 years. The primary causes of an unsatisfactory outcome are due to poor compliance secondary to poor patient and family education, socioeconomic status of the family, increased disease activity, neurological complications, intercurrent infections, renal disease, and especially diffuse proliferative glomerulonephritis. Although the overall prognosis is said to be good, SLE is an episodic disease, with flare and remission. The more frequent a patients get flare episode, the prognosis is tend to be worse. In this patient, the AMT condition is a major factor that influence the prognosis, since the therapy agent for AMT is still underdebating and not available, the thrombocytopenia can be persistent, that increasing the possibility to spontaneous bleeding. ${ }^{1-3,9,18-, 21}$

\section{SUMMARY}

A case of Systemic Lupus Erythematosus (SLE) has been reported. SLE with Amegakaryocyte Trombocytopenia (AMT) in children is a rare case. Diagnosis of AMT was determined by bone marrow aspiration test which revealed isolated diminished megakaryocyte activity. The diagnosis of SLE was determined by ACR scorring system for SLE. The patient was administered antibiotics to overcome the febrile neutropenia, and steroid to prevent the SLE progressitivity. The clinical and laboratory parameter should be evaluated periodically in patient with SLE. Although literally the prognosis 
of SLE in children is good, in this patient thrombocytopenia (AMT) is major factor that may worsen the prognosis.

\section{REFERENCE}

1. Lang BA and Silverman ED. A Clinical Overview of Systemic Lupus Erythematosus in Childhood. Pediatr. Rev.1993;14;194-201.

2. Lehman TJA. Systemic lupus erythematosus in children. In: UpToDate, Basow, DS (Ed), UpToDate, Waltham, MA, UpToDate 2008.

3. Gottlieb BS and Ilowite NT. Systemic Lupus Erythematosus in Children and Adolescents. Pediatr. Rev. 2006;27:323-330.

4. Cela et al. Successful Treatment of Amegakaryocytic Thrombocytopenia With Eltrombopag in a Patient With Systemic Lupus Erythematosus (SLE). Clinical Advances in Hematology \& Oncology 2010;8:806-9.

5. Ziakas et al. Suspects In The Tale Of Lupus-Associated Thrombocytopenia. Clinical and Experimental Immunology 2006;145:71-80.

6. Manson and Rahman. Systemic Lupus Erythematosus. Orphanet encyclopedia 2005;11:1-9.

7. Bhatnagar et al. Pancytopenia in Children: Etiological Profil. Journal of Tropical Pediatrics 2005; 51: 236-9.

8. Lown et al. Acquired Amegakaryocytic Thrombocytopenia: Potential Role of Thrombopoietin Receptor Agonists. Clinical Advances in Hematology \& Oncology 2010; 8: 809-12.

9. Fernando and Isenberg. How To Monitor SLE In Routine Clinical Practice. AnnRheumDis 2005; 64: 524-7.
10. Gabay et al. Guidelines For The Management Of Febrile Neutropenia. ClinicalOncology 2010; 1: 115-22.

11. Kanagara S. Management of Febrile Neutropenia. Commun Oncol 2006; 3: 585-91.

12. Macartney et al. Risk Stratification And Febrile Neutropenia. OE 2007;6:43-5.

13. Hughes et al. 2002 Guidelines for the Use of Antimicrobial Agents in Neutropenic Patients with Cancer. CID 2002;34:730-51.

14. D'Cruz DP, Khamashta MA, Hughes GRV . Systemic lupus erythematosus. Lancet 2007; 369: 587-96.

15. Benseler and Silverman. Systemic Lupus Erythematosus.. Rheum Dis Clin Nam 2007; 33:471-98.

16. Chien and Lin. Autoantibodies To dsDNA, RolSSA, and LalSSB In Systemic Lupus Erythematosus. Advances in clinical Chemistry 2003; 37: 129-72.

17. Spahn JD and Kamada AK. Special Considerations in the Use of Glucocorticoids in Children. Pediatr. Rev 1995;16;266-72.

18. King et al. Lupus Nephritis: Prognostic Factors in Children. Pediatrics 1992;89;240-6.

19. T. Stoll, B. Seifert* And D. A. Isenberg. SLICC/ACR Damage Index Is Valid, And Renal And Pulmonary Organ Scores Are Predictors Of Severe Outcome In Patients With Systemic Lupus Erythematosus. British Journal of Rheumatology 1996;35:248-54.

20. Mirzayan M.J, SchmidtandR.E. and Witte T. Prognostic Parameters For flare In Systemic Lupus Erythematosus. Rheumatology 2000;39:1316-9.

21. Mok et al. A Prospectif Study Of Survival And Prognostic Indicator Of Systemic Lupus Erythematosus In A Southern Chinesse Population. Rheumatology 2000; 39 : 399-406 